\title{
A epilepsia, o epiléptico e o trabalho: relações conflitantes
}

\author{
Epilepsy, epileptics, and work: \\ conflicting relations
}

Maria Rosa Silva Sarmento 1

Carlos Minayo-Gomez 2

\footnotetext{
1 Departamento de Medicina Clínica, Universidade Federal Fluminense. Rua Marquês do Paraná 302 , Niterói, RJ 24210-030, Brasil.

2 Centro de Estudos de Saúde do Trabalhador e Ecologia Humana, Escola Nacional de Saúde Pública. Rua Leopoldo Bulhões 1480 , Manguinhos,

Rio de Janeiro, RJ 21045-900, Brasil.
}

Abstract Participation in the workplace by people with epilepsy is conditioned by various interrelated issues concerning epil epsy itself, the epileptic individual, and soci ety's i mplicit cultural codes. In order to analyze how such conflictive partici pation interferes decisi vely in the quality of life of people with epilepsy, a survey was conducted with 339 patients at the University Hospital of Universidade Federal Fluminense. Patients answered questionnaires including level of schooling, professional qualifications, employment situation, frequency, type, and duration of seizures, associated phenomena, and prejudice towards epilepsy. We observed that satisfactory management of seizures is decisive for their success at work. All patients with daily seizures and $9.3 \%$ of those with weekly seizures had never been employed. We conclude that any intervention in this context must include the implementation of multiprofessional and intersectorial therapeutic programs and legislation to protect epileptic patients' rights in a broad effort at demystification of epilepsy.

Key words Epilepsy; Labor Relations; Occupational Health

Resumo A inserção dos portadores de epilepsia no trabal ho vem condicionada por um conjunto defatores interrel aci onados referentes à epil epsia, ao indivíduo epiléptico e aos códigos culturais implícitos na soci edade. A fim de analisar essa inserção conflitiva, que interfere deci si vamente na qualidade de vida do epiléptico, real izou-se uma investi gação com 339 paci entes do Hospital Uni versitário da Universi dade Federal Fluminense. Apli caram-se questionários incluindo: nível educacional, qualificação profissional, situação de emprego, freqüência etipo de crise, duração, fenômenos associ ados e formas de di scri mi nação. Constata-se que o controle das crises é fator deci si vo para o ingresso e permanência no mercado de trabal ho. Todos os pacientes com crises diárias e 9,3\% daquel es com crises semanais nunca exerceram atividade regular remunerada. Intervir nesse quadro supõe: a implementação de programas terapêuticos multiprofissi onai s e intersetoriais, uma legi slação que proteja os di reitos do epiléptico e um amplo investimento no sentido de desmistificar a epilepsia.

Palavras-chave Epilepsia; Relações Trabalhistas; Saúde Ocupacional 
Introdução

O estudo da problemática dos epilépticos no âmbito do trabal ho envolve a análise de situações interligadas e inseparáveis referentes à epilepsia em si, ao indivíduo epiléptico e ao comportamento do próprio mercado de trabaIho.

As diversas formas de epilepsia, com suas múltiplas etiologias, têm em comum a crise epiléptica e o estigma. Embora a epilepsia possa ser contestada como entidade clínica definida - uma vez que alguns autores a consideram uma síndrome (Browne \& Feldman, 1983; Niedermeyer, 1990) -, seu caráter estigmatizante é indubitavelmente por todos reconhecido e jamais questionado (Ryan et al., 1980; Scambler \& Hopkins, 1980; Scambler, 1987; Collings, 1990b).

A crise epiléptica torna o paciente dependente de terceiros, incapaz de gerenciar sua vida e, apesar de transitória, gera insegurança, facilitando a dominação, além de explicitar o medo da morte. A imprevisibilidade das crises diminui a autoconfiança, a autonomia e a sensação de liberdade. Estas características da doença alteram a individualidade do epiléptico, causam-Ihe alterações psíquicas e dificultam o relacionamento consigo mesmo e com a sociedade (Ryan et al., 1980; Penry \& Devinsky, 1993).

Ao epiléptico é imputada uma incapacidade e, deste modo, o estigma da epilepsia questão cultural tecida por mitos e crenças reduz suas aspirações de vida, aumentando tanto suas auto-exigências quanto sua autodepreciação.

Uma visão histórica acerca da epilepsia permite constatar a multiplicidade de causas que, em seu cerne, conduzem a diversas formas de exclusão e controle social dos epilépticos, indicando o tipo de sociedade à qual pertencem. Nesse sentido, a investigação dos processos que pautam o ingresso dos epilépticos no mundo do trabal ho e sua manutenção no emprego representa campo privilegiado de pesquisa para elucidar situações de rejeição explícitas ou implícitas.

O trabal ho ocupa lugar central na dinâmica da sociedade moderna. Dessa forma, os controles culturais desfavoráveis ao ajustamento social do epiléptico (Jones, 1965) dificultamIhe o acesso ao mercado de trabalho e, portanto, a ascensão social, contribuindo para seu isolamento e exacerbando ou desencadeando patologias psicossociais.

Se, por um lado, o trabalho constitui fonte geradora de saúde física e mental - com freqüência negada ao epiléptico (Dasgupta et al., 1982; Gloag, 1985; Callagham et al., 1992) -, por outro, é capaz de produzir doenças, não sendo incomum que a própria atividade profissional possa dar origem à epilepsia tanto por agressão direta ao cérebro (Allister et al., 1981; Littorin et al., 1988) quanto indireta (Ross, 1988), como também por ser fator desencadeante de crises epilépticas (Winget et al., 1978) em função de determinadas características do processo e organização do trabalho.

No dia-a-dia pode-se observar a relevância prática da questão do trabalho no insucesso terapêutico da epilepsia, na baixa qualidade de vida dos epilépticos e nos obstáculos à integração social.

No Brasil, entretanto, depara-se com a carência de investigações a respeito dos epilépticos em sua interface com o trabal ho. Porém, mesmo no plano internacional, há insuficiência de informações quanto ao número de epilépticos em idade produtiva e ao de desempregados (Allister et al., 1981; Dasgupta, 1992).

\section{O mercado de trabalho e os epilépticos}

O acesso a um mercado de trabalho cada vez mais competitivo, agravado pelo crescente nível de desemprego, coloca as pessoas com algum tipo de desabilidade em posição desvantajosa, mesmo que estejam profissionalmente capacitadas para o desempenho da atividade pleiteada e que suas limitações, por si só, não constituam impedimento ao exercício das funções propostas.

O temor da crise, de sua imprevisibilidade e do estigma transformam o epiléptico em pessoa medrosa, insegura e ansiosa, conduzindoo à acomodação que o inibe na luta pelos seus direitos.

Vários são os motivos freqüentemente alegados por alguns empregadores ao preterirem os epilépticos: medo de que as crises causem acidentes, em especial, na indústria; suposição de que os epilépticos têm menor capacidade de trabalho e, por conseguinte, baixa produtividade; crença de que o absenteísmo é maior entre epilépticos em virtude das crises; receio de que as crises epilépticas no local de trabalho gerem intranqüilidade nos demais empregados e que a produtividade diminua. Em última instância, é o peso do estigma que se faz presente, nesses casos, no momento de decidir a contratação e a demissão dos epilépticos (Scambler \& Hopkins, 1980; Cooper, 1995).

A justificativa do absenteísmo em decorrência de crises epilépticas e suas conseqüências carece de melhor fundamentação. Dasgupta et al. (1982) demonstram, inclusive, que 
o de curta duração é menor entre trabal hadores epilépticos, embora tenham encontrado os afastamentos mais longos nesse grupo.

No que se refere à capaci dade para o trabaIho, Callagham et al. (1992) observam que esta é normal nos pacientes cujas únicas manifestações clínicas dizem respeito à epilepsia, sem que haja outra doença associada. Nesse mesmo sentido, Lassow et al. (1997) - ao comparar educação, desempenho profissional, absenteísmo e salário em trabalhadores epilépticos e não-epilépticos com ocupações semel hantes encontraram a menor remuneração dos epilépticos como única diferença estatisticamente significativa entre ambos os grupos. Espir et al. (1991), no entanto, reconhecem que - apesar de não ter sido possível encontrar diferenças, sob esse aspecto, entre trabalhadores epilépticos e portadores de outras doenças - os próprios epilépticos se consideram em situação de inferioridade nas oportunidades de emprego e na ascensão profissional, desistindo de competir e limitando suas perspectivas de vida.

Em relação à acidentabilidade alegada, um workshop realizado no International Bureau for Epilepsy, em 1966, concluiu não haver maior risco de acidentes no trabalho em pessoas epilépticas do que na população em geral. Beghi \& Cornaggia (1997) tendem a conclusão semeIhante em estudo de coorte multinacional que vêm realizando em sete países europeus.

Ao lado dessa discriminação velada e não oficial, existe outra, garantida por dispositivos legais que proíbem o exercício de determinadas profissões, sob a argumentação de que a crise epi léptica expõe a riscos de vida não só os pacientes como também outras pessoas. Contudo, alguns autores (Fabing \& Barrow, 1960) chamam a atenção para o caráter discriminatório das leis que regulamentam o trabalho do epiléptico, por julgá-las arcaicas à luz dos conhecimentos atuais e dos avanços terapêuticos no controle das crises. Alertam para suas conseqüências negativas já que, se aplicadas sem discernimento, contribuem para dificultar o ajuste social, aumentar a discriminação e diminuir a aderência ao tratamento. Diante desse quadro, Beresford (1988) aponta a necessidade de revisar a legislação existente para garantir maior proteção aos epilépticos contra a segregação infundada. O temor das restrições, oficiais ou não, acaba por induzir os epilépticos a ocultar sua condição na fase de seleção para o emprego.

É importante ressaltar que, com o tratamento medicamentoso, $75 \%$ dos pacientes ficam assintomáticos e cerca de $5 \%$ têm crises eventuais. Portanto, a barreira das crises na conquista e manutenção do emprego vem-se desmoronando, apesar de que os efeitos colaterais das drogas antiepilépticas (DAE), principalmente a sonolência, possam constituir restrição a determinadas atividades profissionais (Udel, 1960). Tomando em conta que $80 \%$ dos epilépticos são estáveis, o acesso ao trabal ho e à manutenção do emprego dependem, quase que exclusivamente, dos critérios dos empregadores que, em sua maioria, vêm pautados pelo preconceito. A preocupação de manter o emprego, mesmo para estes pacientes, transforma-se em motivo de ansiedade, gerando estresse, angústia e depressão, fatores desencadeantes de crises, as quais aumentam, por sua vez, o medo da demissão, o que ocorre principalmente após crise no local de trabalho. Instaura-se assim um circuito altamente nocivo, permeado, em essência, pelo estigma.

A situação mais drástica corresponde, porém, à dos $20 \%$ de epilépticos que não têm o controle de suas crises e, em conseqüência, vêm muito mais reduzidas as chances de sucesso no mundo trabalho.

Algumas profissões - listadas na Tabela 1 devem ser evitadas pelos epilépticos. Quanto a babás, Niedermeyer (1990) chama a atenção para a inexistência de conseqüências sérias no trabalho. Em nossa prática profissional, constatam-se situações semelhantes entre enfermeiras, vigias e aeromoças sem crises epilépticas. Em razão das peculiaridades da epilepsia que não evolui de maneira linear, em que a crise tem caráter episódico e apresentando remissões e exacerbações - é preciso distinguir entre os pacientes estáveis, porém em uso de DAE, os assintomáticos sem uso de medicação e os

Tabela 1

O cupações e atividades consideradas impróprias para epilépticos.

Policiais

Bombeiros

Vigias solitários

Instrutor de natação e salva-vidas

Babás

Enfermagem

Cirurgia

Dirigir veículos motorizados

Controle de máquinas e/ou equipamentos

Serviços militares

Trabalhos em altitude ou com uso de escadas

Modificado de Niedermeyer, 1990. 
que continuam a ter crises. Por isso, restrições devem ser feitas com reservas. Para os pacientes cujas crises não estão controladas, cabe aplicar as regras gerais existentes, enquanto, nos demais casos, as limitações profissionais exigem constante reavaliação. As únicas restrições dizem respeito àquelas ocupações que colocam em situação de risco a sua vida e a de outras pessoas. Uma vez que a terapia medicamentosa resgatou socialmente o epiléptico, não procede manter a rigidez de legislações capazes de conduzir previamente a uma discriminação no trabalho.

O custo do epiléptico aos cofres públicos tem propiciado estudos - como o de Cockerell et al. (1994), no Reino Unido - que demonstraram ser de $f 1.239$ milhões o gasto anual com o desemprego dos epilépticos, o que eqüivale a $f$ 2.887 por paciente. Tal fato tem levado o Estado a proibir, por meio de leis, a discriminação de trabalhadores epilépticos (Beresford, 1988) e a promover programas de reabilitação profissional que propiciem adequada reintegração no mercado de trabal ho em funções compatíveis com a epilepsia. No Brasil, carece-se de estimativas quanto a esse custo, como também de política de saúde para os epilépticos.

Os epilépticos, em razão das restrições de emprego - em particular, para aqueles dos extratos populares - recorrem habitual mente à economia informal e ao subemprego, quando não permanecem na dependência econômica da família, dos cofres públicos ou da mendicância, o que contribui para aumentar o estigma da doença e diminuir a auto-estima dos pacientes, além de engrossar a ampla parcela de trabalhadores marcados pela exclusão social e pela vulnerabilidade econômica.

É provável que, em nenhuma outra situação, as características estigmatizantes da epilepsia se tornem tão explícitas e exerçam ação tão avassaladora na vida do epiléptico como em seus confrontos no âmbito do trabalho. As restrições na escolha da profissão, o sentimento de isolamento e de discriminação, exacerbado pelas dificuldades de obtenção e de manutenção do emprego, dificultam a aderência ao tratamento e contribuem para o insucesso terapêutico no controle das crises epilépticas, fechando um ciclo danoso ao paciente, o qual, além de impor-Ihe dependência econômica, interfere em sua qualidade de vida.
Análise da relação epilepsia-epilépticotrabalho no Hospital Universitário Antônio Pedro

Neste estudo descritivo a respeito da inter-relação epilepsia - epiléptico - trabalho - realizado com pacientes do Setor de Epilepsia - SE do Hospital Universitário Antônio Pedro - HUAP da Universidade Federal Fluminense - UFF teve-se o propósito principal de analisar as questões implicadas na relação conflituosa do epiléptico com o trabalho, a fim de que esse problema - relevante no quotidiano do epiléptico - seja levado em consideração nos programas de atenção à saúde dos epilépticos que pretendam assegurar o estabelecimento de condutas voltadas, em seu conjunto, à melhoria da qualidade de vida desses pacientes.

\section{Pacientes e métodos}

Para estudar a ação da epilepsia e a ingerência dos fatores vinculados à condição de epiléptico na vida profissional, foi elaborado um roteiro de entrevista, com questões fechadas e abertas, que permitisse descrever a relevância dessa problemática sob o ponto de vista dos pacientes. Esse roteiro foi testado previamente, a fim de evitar a omissão de questões significativas. Para sua elaboração e validação, não foram encontrados subsídios, na bibliografia a que se teve acesso, que contemplassem as distinções e correlações estabelecidas nesta pesquisa. No roteiro, foram abordados os seguintes temas: características da crise - freqüência, tipo, duração, fenômenos associados - e o tempo de recuperação do paciente; nível educacional e qualificação profissional; situação laboral - empregado no mercado formal ou informal, desempregado ou sem ativi dade remunerada regular - e as manifestações de discriminação - demitido em conseqüência das crises e/ ou preterido na ascensão funcional -, bem como os fatores limitantes para o acesso ao emprego e sua manutenção.

Dessa forma, foi possível estabelecer a correlação entre as diversas experiências vividas pelo epiléptico no acesso ao trabalho ou na trajetória laboral com os dados objetivos da epilepsia: os eventos da crise epiléptica e os sintomas que a sucedem antes da recuperação.

Dos 398 pacientes que compareceram ao SE do HUAP entre fevereiro de 1995 e julho de 1996, foram selecionados 339, de acordo com três critérios básicos de inclusão: o diagnóstico de certeza da epilepsia, segundo os critérios da Internati onal League Against Epilepsy - ILAE (Comission on Classification and Terminology 
of the International League Against Epilepsy, 1989); a idade igual ou superior a 18 anos; e, por fim, a participação em mais de uma entrevista. Excluíram-se os que tinham retardo mental, lesões estruturais cerebrais conhecidas ou doença concomitante. Os 339 pacientes foram entrevistados individualmente, pelo menos duas vezes, durante as consultas para complementar ou confirmar determinadas informações.

Avaliou-se objetivamente a epilepsia, estudando as crises epilépticas mediante informações diárias, anotadas em agendas pelos pacientes ou seus familiares, com relação ao tipo de crise, a duração, a freqüência e os fenômenos associados. Para identificar as crises, adotou-se uma classificação simplificada da ILAE (Comission on Classification and Terminology of the International League Against Epilepsy, 1989). Nas Crises Parciais (CP), se a consciência está preservada: Crise Parcial Simples (CPS); com perda da responsividade consciente: Crises Parciais Complexas (CPC); crises parciais seguidas de perda da consciência, com ou sem convulsão: Crises Parciais Secundariamente Generalizadas (CPSG); crises com perda da consciência desde o início da crise: Crises Generalizadas (CG). Dentre as CG, consideraramse as crises com associação de atividade tônico-clônica: Crises Tônico-Clônicas (CTC); com mioclonias, Crises Mioclônicas (CM). Denominou-se "Outras Crises" (OC) os demais tipos de crises generalizadas. As crises não incluídas nessa classificação são as Não Classificadas (CNC) e as com mais de um tipo de crise, as Crises Mistas (CMi).

No período imediatamente após a crise até a recuperação do paciente, analisou-se a sintomatologia a partir de uma listagem que foi elaborada em ordem de freqüência e relevância, conferidas pelos pacientes, enquanto causa de aumento das desabilidades para o trabalho.

Final mente foram identificados os fatores reconhecidos pelos pacientes como limitantes para o acesso ao emprego e sua manutenção, estimados como peculiares a todos os trabaIhadores epilépticos.

\section{Resultados}

Dentre os entrevistados, $48 \%$ eram do sexo masculino, 27,6\% eram analfabetos, 0,6\% tinham curso superior e $98,4 \%$ não tinham qualquer qualificação profissional. O número de epilépticos empregados foi de $42,3 \%$, dos quais apenas $15,7 \%$ se encontravam no mercado formal de trabalho.

Todos omitiram a condição de epiléptico com medo de que o estigma de sua história patológica tivesse peso maior que seus méritos profissionais fosse por experiência pessoal, em ocasiões anteriores, ou de terceiros. Dos entrevistados, 1,3\% ocultaram a epilepsia e desenvolviam atividades profissionais consideradas indevidas para os epilépticos.

Ao relacionar a freqüência das crises com a situação de emprego (Tabela 2), constatase que, dos 52,9\% pacientes estáveis, ou seja, com freqüência anual de crise, $40,3 \%$ estavam empregados, embora a grande maioria no mercado informal. Todos os pacientes com crises diárias e semanais encontravam-se desempregados, bem como $94,6 \%$ daqueles com crises mensais. Os pacientes com crises diárias e 9,3\% dos que apresentavam crises semanais jamais haviam exercido atividade regular remunerada.

Dentre os desempregados, $23,6 \%$ nunca exerceram atividades lucrativas com regularidade. Do total de pacientes, $88,3 \%$ já foram discriminados: $38,6 \%$ demitidos em decorrência de crises e $49,7 \%$ preteridos na ascensão funcional (Tabela 3).

Como se pode deduzir da Tabela 4, a maior proporção de pacientes desempregados ocorreu nos portadores de crises mistas, $84,2 \%$, se-

Tabela 2

Freqüência das crises e situação de emprego na população estudada.

\begin{tabular}{|c|c|c|c|c|c|c|c|}
\hline \multirow{3}{*}{$\begin{array}{l}\text { Freqüência das } \\
\text { crises ( } n=339)\end{array}$} & \multirow{3}{*}{$\begin{array}{c}\text { Pacientes } \\
n\end{array}$} & \multicolumn{6}{|c|}{ Situação de emprego } \\
\hline & & \multicolumn{2}{|c|}{ Emprego formal } & \multicolumn{2}{|c|}{ Emprego informal } & \multicolumn{2}{|c|}{ Desemprego } \\
\hline & & $n$ & $\%$ & $n$ & $\%$ & $\mathrm{n}$ & $\%$ \\
\hline Diária & 6 & - & - & - & - & 6 & 1,8 \\
\hline Semanal & 42 & - & - & - & - & 42 & 12,3 \\
\hline Mensal & 111 & 3 & 1,0 & 3 & 1,0 & 105 & 31,0 \\
\hline Anual & 180 & 50 & 14,7 & 87 & 25,6 & 43 & 12,6 \\
\hline
\end{tabular}


Tabela 3

Freqüência das crises e discriminação no trabalho na população estudada.

\begin{tabular}{|c|c|c|c|c|c|}
\hline \multirow{3}{*}{$\begin{array}{l}\text { Freqüência das } \\
\text { crises }(n=299)\end{array}$} & \multirow{3}{*}{$\begin{array}{c}\text { Pacientes } \\
n\end{array}$} & \multicolumn{4}{|c|}{ Formas de discriminação } \\
\hline & & \multicolumn{2}{|c|}{ Demitidos por crises } & \multicolumn{2}{|c|}{ Preteridos por serem epiléticos } \\
\hline & & $n$ & $\%$ & $n$ & $\%$ \\
\hline Diária & 6 & - & - & 6 & 1,8 \\
\hline Semanal & 63 & 28 & 8,3 & 35 & 10,3 \\
\hline Mensal & 164 & 83 & 24,5 & 81 & 24,0 \\
\hline Anual & 66 & 20 & 5,8 & 46 & 13,6 \\
\hline
\end{tabular}

Tabela 4

Tipo de crise e situação de emprego na população estudada.

\begin{tabular}{|c|c|c|c|c|c|c|c|}
\hline \multirow{3}{*}{$\begin{array}{l}\text { Tipo de crise } \\
(n=339)\end{array}$} & \multirow{3}{*}{$\begin{array}{c}\text { Pacientes } \\
n\end{array}$} & \multicolumn{6}{|c|}{ Situação de emprego } \\
\hline & & \multicolumn{2}{|c|}{ Emprego formal } & \multicolumn{2}{|c|}{ Emprego informal } & \multicolumn{2}{|c|}{ Desempreg } \\
\hline & & $n$ & $\%$ & $n$ & $\%$ & $n$ & $\%$ \\
\hline$C P$ & 75 & 22 & 6,6 & 17 & 5,0 & 36 & 10,6 \\
\hline CPSG & 91 & 12 & 3,5 & 19 & 5,8 & 60 & 17,8 \\
\hline CG & 131 & 15 & 4,5 & 48 & 14,1 & 68 & 20,0 \\
\hline$\tilde{N}$. Clas. & 4 & 4 & 1,1 & - & - & - & - \\
\hline $\mathrm{CMi}$ & 38 & - & - & 6 & 1,7 & 32 & 9,3 \\
\hline
\end{tabular}

CP: Crises Parciais; CPSG : Crises Parciais Secundariamente Generalizadas; CG: Crises Generalizadas;

N. Clas.: Não Classificadas; CM i: Crises Mistas.

guidos pelos epilépticos com crises parciais secundariamente general izadas, cujo desemprego atingiu $65,9 \%$. Foi ainda nos pacientes com crises mistas que se verificou a maior percentagem (34,7\%) dos que não haviam conseguido ingressar no mercado.

Ao comparar o número absoluto de pacientes por tipo de crise (Tabela 4) e o de discriminados (Tabela 5), nota-se que o maior percentual de demitidos em razão da crise $(63,2 \%)$ foi observado também entre pacientes com crises mistas, ao passo que os portadores de crises parciais secundariamente generalizadas foram os mais preteridos por sua condição de epilépticos $(84,6 \%)$, seguindo-se aqueles com crises mistas (60,5\%)

A Tabela 6 mostra que, dentre os pacientes supostamente demitidos por causa do tempo de duração da crise, a maior proporção encontrada foi entre os que tiveram crises com duração entre 30 e 59 segundos. As demissões nos casos de absenteísmo, em virtude da intensidade dos fenômenos associados - listados na Tabela 7 por ordem da relevância conferida pelos pacientes - ocorreram com maior freqüência quando a falta ao emprego foi superior a um dia.
Não foi possível estabelecer correlação entre a manutenção do emprego e os sintomas pós-ictais, uma vez que este eram percebidos pelos epilépticos como componentes da própria sintomatologia da crise que motivou a demissão, ao contribuírem para aumentar as dificuldades no trabalho.

Estes dados foram desconsiderados, já que apenas $28,6 \%$ dos pacientes conseguiram especificar que a demissão deveu-se ao tempo prolongado para sua recuperação.

No entanto, mesmo na ausência do nexo com o emprego, pode-se concluir que a sintomatologia após a crise epiléptica (Tabela 7) influiu, de algum modo, para aumentar o insucesso profissional e o desajuste social dos pacientes.

Cabem aqui algumas considerações emanadas da prática clínica acerca desses dados. A sonolência, as alterações de memória e a irritabilidade, apontadas como os sintomas mais freqüentes em todas as crises, ocorreram principalmente naquelas em que a consciência não estava preservada. Quanto mais prolongadas, maior era a situação de desconforto e constrangimento dos pacientes ao recuperar-se da 
Tabela 5

Tipo de crise e discriminação no emprego na população estudada.

\begin{tabular}{|c|c|c|c|c|c|}
\hline \multirow{3}{*}{$\begin{array}{l}\text { Tipo de crise } \\
(n=299)\end{array}$} & \multirow{3}{*}{$\begin{array}{c}\text { Pacientes } \\
n\end{array}$} & \multicolumn{4}{|c|}{ Formas de discriminação } \\
\hline & & \multicolumn{2}{|c|}{ Demitidos por crises } & \multicolumn{2}{|c|}{ Preterid os por serem epiléticos } \\
\hline & & $\mathrm{n}$ & $\%$ & $n$ & $\%$ \\
\hline $\mathrm{CP}$ & 37 & 27 & 7,8 & 10 & 3,1 \\
\hline CPSG & 123 & 46 & 13,6 & 77 & 22,6 \\
\hline CG & 92 & 34 & 10,1 & 58 & 17,2 \\
\hline Cmi & 47 & 24 & 7,1 & 23 & 6,8 \\
\hline
\end{tabular}

CP: Crises Parciais; CPSG : Crises Parciais Secundariamente Generalizadas; CG: Crises Generalizadas;

CMi: Crises Mistas.

crise. Os últimos três sintomas, apesar da menor freqüência, traziam complicações significativas, principalmente os automatismos deambulatórios - habitualmente confundidos com sintomatologia de doença mental -, bem como os distúrbios da fala e as dificuldades motoras, tidos como muito graves e objeto de cuidados médicos imediatos.

Finalmente, os próprios pacientes relataram fatores de natureza diversa identificados por eles como peculiares a sua condição de epilépticos e conhecidos como "epilepsia-relacionados", que dificultam o ingresso no mercado de trabalho, a manutenção do emprego e a ascensão profissional (Tabela 8). A falta de qualificação profissional e o estigma foram percebidos como os maiores entraves à obtenção de emprego, razão pela qual usavam a tática de omitir sua condição. O estigma foi ainda apontado como a principal causa de perda do emprego. A inclusão, entre outros fatores descritos, da dependência do médico e do medicamento foi ressaltada sobretudo pela dificuldade em conseguir a DAE, só obtida por intermédio de receituário controlado, o que determinou a ausência ao trabalho e a demissão.

\section{Discussão e conclusões}

Inicialmente, convém ressaltar que os diversos fatores utilizados neste estudo para configurar a relação epilepsia-epiléptico-trabalho estão imbricados, são interdependentes e interagem entre si. No entanto, essa constatação de caráter geral não exclui a necessidade de levar em consideração a individualidade do epiléptico e a especificidade de cada caso.

É oportuno lembrar ainda que os pacientes do SE do HUAP provêm de população de baixa renda e sem qualificação profissional. Parte considerável desses pacientes era analfabeta. Os
Tabela 6

Demissão do emprego segundo a duração da crise epiléptica e absenteísmo.

\begin{tabular}{|c|c|c|c|}
\hline \multirow[t]{2}{*}{ Pacientes epilépticos } & \multirow[t]{2}{*}{ Total } & \multicolumn{2}{|c|}{ Demitidos } \\
\hline & & $n$ & $\%$ \\
\hline \multicolumn{4}{|l|}{$\begin{array}{l}\text { Duração da crise } \\
\text { epiléptica }(n=339)\end{array}$} \\
\hline$<30$ segundos & 282 & 85 & 30,1 \\
\hline 30 a 59 segundos & 42 & 35 & 83,3 \\
\hline 60 segundos ou mais & 15 & 11 & 73,3 \\
\hline \multicolumn{4}{|l|}{$\begin{array}{l}\text { Absenteísmo } \\
\text { no trabalho }(n=106)\end{array}$} \\
\hline $1 \mathrm{dia}$ & 35 & 21 & 60,0 \\
\hline 2 dias ou mais & 71 & 53 & 74,6 \\
\hline
\end{tabular}

resultados obtidos, portanto, refletem predominantemente as características de uma população sem grandes perspectivas de integrar o mercado formal de trabalho. A condição de epiléptico vem agravar tal quadro, podendo configurar uma justificativa a mais de exclusão social. A carência de dados estatísticos acerca da epilepsia e da ocupação no Brasil, aliada à inexistência de estudos afins no país, impede o estabelecimento de comparações. Trata-se, no entanto, de problemática universal; assim, conquanto cientes das diferenciações próprias de cada sociedade, analisou-se a população estudada à luz das pesquisas internacionais referentes ao assunto.

Partiu-se do pressuposto que o trabalho confere ao indivíduo um status pessoal e social que contribui na estruturação de sua identidade. O epiléptico, por sua relação conflitante com o mundo do trabalho, freqüentemente é levado a renunciar a esse direito, o que lhe traz repercussões adversas na vida cotidiana, afetando sua saúde física e mental. Nesse sentido, é revelador que maiores índices de desempre- 
Tabela 7

Sintomas após as crises epilépticas.

\begin{tabular}{lc}
\hline Sintoma & $\%$ \\
\hline Sono & 90 \\
Alterações da memória & 90 \\
Irritabilidade & 90 \\
Alterações do humor & 85 \\
Cefaléia & 80 \\
Dores Musculares & 75 \\
Distúrbios da fala & 60 \\
Automatismo & 60 \\
Déficit Motor & 5 \\
\hline
\end{tabular}

Tabela 8

Fatores limitantes do epiléptico em relação ao trabalho.

\begin{tabular}{lr}
\hline Fator & $\%$ \\
\hline Falta de qualificação profissional & 100 \\
Estigma & 100 \\
Imprevisibilidade das crises & 95 \\
Medo das crises & 95 \\
Insegurança & 90 \\
Baixa de auto-estima & 85 \\
Dificuldades de relacionamento & 80 \\
Vergonha de ser epiléptico & 80 \\
Dependência: família, médico & 75 \\
e da medicação & \\
Irritabilidade & 70 \\
Alterações de Humor & 70 \\
\hline
\end{tabular}

go, se comparados aos da população, em geral, em idade produtiva, sejam encontrados entre os epilépticos, conforme observado em diversos estudos (Rodin, 1972, 1982; Collings, 1990a) e plenamente evidenciado nesta investigação. Ser epiléptico representa empecilho adicional para obter emprego, quando estar empregado constituiria um preditor de boa qualidade de vida (Collings, 1990b).

Alguns autores destacam a predominância do desemprego entre os epilépticos com crises freqüentes (Elwes et al., 1991; Dasgupta, 1992) e, sobretudo, nos de baixa renda (Scambler \& Hopkins, 1980; Gloag, 1985). Neste estudo, o desemprego atingiu a totalidade dos pacientes com crises diárias e semanais, tal como 93,9\% daqueles com crises mensais.
Os resultados demonstram que o tipo de crise é igualmente fator diferenciador em relação ao emprego, identificando o maior percentual de desempregados no grupo dos portadores de crises mistas.

A constatação de que o peso do estigma representa uma das maiores barreiras ao ingresso e à permanência no mercado de trabal ho foi unânime na opinião dos pacientes e é consensual na literatura. Jacoby (1962) afirma que o estigma afetou $32 \%$ de seus pacientes, mesmo não tendo encontrado diferença significativa entre as pessoas com epilepsia ativa e as estáveis. Tettenborn \& Krämer (1992), em investigação realizada no Reino Unido, enfatizam as dificuldades do epiléptico no acesso ao emprego decorrentes de problemas socioculturais. Collings (1990b) é ainda mais incisivo. Para o autor, embora a freqüência das crises seja relevante, o estereótipo negativo da epilepsia é o componente mais restritivo e perpassa, inclusive, o diagnóstico da epilepsia. Scambler (1987) acrescenta outros fatores limitantes, referidos, no atual estudo, a essas desvantagens do epiléptico: a falta de qualificação profissional e a baixa da auto-estima.

A discriminação no emprego - que nos dados foi observada, em particular, em pacientes com crises mais freqüentes e com crises mistas ou parciais secundariamente generalizadas - é relatada em 35\% dos pacientes por Chaplin et al. (1998), atingindo 59\% daqueles com crises não controladas. Da mesma forma, Ryan et al . (1980) referem experiências de discriminação em $46 \%$ dos epilépticos de sua amostra, sendo que $22 \%$ tinham sido demitidos dos últimos quatro empregos por fatores relacionados à epilepsia. Elwes et al. (1991) citam que $42 \%$ de seus pacientes foram vitimados pelo estigma da epilepsia, em muitos casos pelo que qualificam de "discriminação legitimada". Quanto à preterição dos empregados em suas carreiras por problemas relacionados à epilepsia - verificada, na presente investigação, em $49,7 \%$ dos pacientes Scambler \& Hopkins (1980) revelam ter ocorrido em $42 \%$ dos epilépticos de sua amostra.

Nesta casuística, todos os pacientes empregados ocultaram a condição de epiléptico pelo medo do estigma, o que foi notado ainda por vários pesquisadores, porém não de modo tão contundente. Jones (1965) refere que $26 \%$ dos epilépticos empregados na Inglaterra ocultaram sua enfermidade. Scambler \& Hopkins (1980) descrevem que, dos 53\% de seus pacientes empregados, $25 \%$ também a omitiram e $28 \%$ só a revelaram após ter obtido o emprego.

A omissão da doença certamente representa estratégia de defesa compreensível frente à 
possibilidade real de recusa no exame admissional, dadas as limitadas chances que uma população com semelhantes características socioeconômicas tem de ingressar em mercado de trabalho al tamente competitivo. Cabe, porém, orientar os pacientes para que avaliem a oportunidade de revelar sua condição, consideradas as restrições ocupacionais já referi das, após demonstrada sua capacidade laborativa. $\mathrm{O}$ esclarecimento, em situações favoráveis à continuidade no emprego, contribui para aumentar a segurança e a auto-estima, ao mesmo tempo em que diminui o estresse e a ansiedade, fatores prejudiciais ao rendimento profissional e potencialmente deflagradores de crises.

O controle das crises é fator decisivo à obtenção e manutenção do emprego, como apontado em outros trabalhos (McLellan, 1987; Floyd et al., 1992; Perrine, 1993; Jacoby, 1995). Estefato também foi detectado neste estudo. Apesar da causalidade múltipla, a discriminação dos epilépticos no trabalho guarda íntima relação com a freqüência das crises. Neste estudo, 95,3\% dos pacientes estáveis encontravam-se empregados, ainda que a maioria no mercado informal.

A falta de orientação profissional e, principalmente, de qualificação profissional - fator limitante reconhecido por todos os pacientes gera problemas psicossociais, agravando o desemprego e dificultando o ajuste social (Olsson \& Campenhausen, 1993). Os obstáculos do epiléptico no acesso ao mercado de trabalho e na manutenção do emprego representam entrave a mais na aderência ao tratamento.

Tal situação remete à necessidade de esclarecer o paciente acerca da escolha da profissão - tomando em conta habilidades, capacidades e limitações - e, ainda, de alertá-lo quanto ao exercício de al guns tipos de trabal ho que possam pôr em risco sua vida e a de outras pessoas. Dessa forma, evita-se criar expectativas fora da realidade do epiléptico que venham a contribuir para aumentar suas frustrações. Caberia aos programas de atenção à saúde do trabal hador, governamentais ou não, incumbirem-se da importante tarefa de acompanhar o desempenho profissional desses pacientes, orientandoos de modo a tornar mais efetivo o controle das crises e também possibilitar que sejam inseridos em funções compatíveis com suas peculiaridades. Collings (1990b) chama a atenção para o fato de que as desabilidades podem ser prevenidas, se as percepções dos epilépticos e suas circunstâncias forem valorizadas. O diagnóstico, mediante exames médicos e psicológicos, efetuado sob essas premissas, permite inclusive corrigir deficiências passíveis de tratamento.
Nessa perspectiva, Baldwin (1960) e, posteriormente, Espir et al. (1991) destacam o papel dos centros de saúde ocupacional no desempenho do trabalhador epiléptico, orientando-o quanto ao controle das crises e à procura de alternativas de regime e condições de trabaIho. Espir et al. (1991) referem ainda que, dos 50.000 novos empregados anualmente, $20 \%$ foram encaminhados ao Civil Occupational Health Service, em Londres, para a emissão de pareceres relativos a questões correlatas às crises epilépticas e ao emprego.

Considerou-se que um programa terapêutico eficiente deve ter como centro o epiléptico e, a partir dele, a epilepsia. Dada a amplitude das questões envolvidas, um programa dessa natureza requer a incorporação de profissionais de diversas formações e a articulação com vários outros setores, desde os serviços de saúde ocupacional, de assistência médica especializada e de reabilitação até a assessoria jurídica.

Assim como outros autores (Jones, 1965; Long \& Moore, 1979; Kettle, 1984), acredita-se que esse propósito só pode ser alcançado com a implementação de uma política de saúde para os epilépticos consolidada a partir de amplo espectro de condutas capazes de dirimir dúvidas e desmistificar a epilepsia, ao mesmo tempo em que vise preparar o epiléptico para o ingresso e permanência no mercado de trabal ho, potencializando suas aptidões e respeitando seus limites. Nessa direção, é ilustrativo o estudo de Hicks \& Hicks (1991) que descrevem os avanços obtidos na conquista e preservação do emprego dos epilépticos por meio de investimento pedagógico realizado em São Francisco, ao longo de 30 anos, junto aos empregadores e a diversos setores sociais.

Em síntese, à luz dos dados obtidos, podese inferir que o insucesso profissional do epiléptico decorre da conjugação de condições inerentes à epilepsia, ao epiléptico e dos códigos culturais implícitos na sociedade em mercado de trabalho altamente competitivo. No entanto, apesar da importância, da complexidade e das conseqüências dessas relações conflitantes, os profissionais de saúde, os governantes e os legisladores vêm negligenciando a questão.

A ausência, no Brasil, de legislação que proteja os epilépticos e regulamente seus direitos, os expõe a discriminação prévia. No âmbito dos serviços de saúde constata-se também a carência de programas terapêuticos que contemplem concomitantemente o controle da doença e o investimento em um conjunto de ações educativas dirigidas aos epilépticos e 
seus familiares, aos empregadores e à sociedade em geral.

Finalmente espera-se que as situações reveladas neste estudo não se perpetuem e sirvam de subsídio para avançar no longo, mas impreterível, percurso de construção de políticas sociais emancipatórias.

\section{Referências}

ALLISTER, C.; LUSH, M. \& OLIVER, J. S., 1981. Status epilepticus caused by solvent abuse. BMJ, 283: 1156.

BALDWIN, M., 1960. The problem of temporal lobe epilepsy in occupational medicine. Archives of Environmental Health, 1:87-90.

BEGHI, E. \& CORNAGGIA, C., 1997. Epilepsy and every life risk. A case referent study: Rationale, study design and preliminary results. Risk in Epilepsy Study Group. Journal of Neuroepidemiology, 16:207-216.

BERESFORD, H. R., 1988. Legal implications of epilepsy. Epilepsia, 29:S114-S121.

BROWNE, T. R. \& FELDMAN, R. G., 1983. Epilepsy: An overview. In: Epilepsy: Diagnosis and Management (T. R. Browne \& R. G. Feldman, eds.), pp. 110, Boston: Library of Congress Catalog Card.

CALLAGHAN, N.; CROWLEY, M. \& GOGGIN, T., 1992. Epilepsy and employment, education and social status. Irish Medical Journal, 85:17-19.

CHAPLIN, J. E.; WESTER, A. \&TOMSON, T., 1998. Factors associated with employment problems of people with established epilepsy. Seizure, 7:299-303.

COCKERELL, C.; HART, Y. M.; SANDER, J. W. S. \& SHORVON, S. D., 1994. The cost of epilepsy in the U.K.: An estimation based on results from two population-based studies. Epilepsy Research, 18:249260.

COLLINGS, J., 1990a. Psychossocial well-being and epilepsy: An empirical study. Epilepsia, 31:418-26.

COLLINGS, J., 1990b. Epilepsy and well-being. Social Science \& Medicine, 31:165-170.

COMISSION ON CLASSIFICATION AND TERMINOLOGY OF THE INTERNATIONAL LEAGUE AGAINST EPILEPSY, 1989. Proposal for revised clinical classification of epileptisies and epileptic syndromes. Epilepsia, 30:389-399.

COOPER, M., 1995. Epilepsy and employment - Employer' s attitudes. Seizure, 4:193-199.

DASGUPTA, A. K., 1992. Epilepsy and employability: A comparative evaluation of epileptic employees working in the steel plants of India. Journal of Occupational Medicine, 42:137-142.

DASGUPTA, A. K.; SAUNDERS, M. \& DICK, D., 1982. Epilepsy in the British Steel Corporation: An evaluation of sickness, accident and work records. British Journal of Industrial Medicine, 39:145-148.
ELWES, R. D. C.; M ARSHALL, J.; BEATTIE, A. \& NEWMAN, P. K., 1991. Epilepsy and employment. A community based survey in na area of high unemployment. Journal of Neurology, Neurosurgery and Psychiatry, 54:200-203.

ESPIR, M.; FLOYD, M. \& CHAPLIN, J., 1991. Occupational aspects of epilepsy in the civil service. British Journal of Industrial Medicine, 48:665-669.

FABING, H. \& BARROW, R. L., 1960. Epilepsy and the law. Special Committee on Epilepsy Legislation of the American League Against Epilepsy. Archives of Environmental Health, 1:91-98.

FLOYD, M. D., 1992. A review of published in epilepsy and employment. In: A Medical Symposium on Current Problems and Best Pratices (F. Edwards, M. Espir \&J. Oxley, eds.), pp. 3-7, London: Royal Society of Medicine Ltd.

GLOAG, D., 1985. Epilepsy and employment. British Medical Journal, 291:2-3.

HICKS, R. A. \& HICKS, M. J., 1991. Attitudes of major employers toward the employment of people with epilepsy: A 30 - year study. Epilepsia, 32:86-88.

JACOBY, A., 1992. Epilepsy and the quality of everyday life: Findings from a study of people with well-controlled epilepsy. Social Science $\&$ Medicine, 34:657-666.

JACOBY, A., 1995. Impact of epilepsy on employment status: Findings from a UK study of people with well-controled epilepsy. Epilepsy Research, 21: 125-132.

JONES, J., 1965. Employment of epileptics. Lancet, ii: 486-489.

JOUANNE, T.; MUH, P. \& AUBET, A., 1991. Communications. Epilepsie et travaux dangereux. L'experience des consultations de pathologie professionnelle de Reenes. Archives de Maladie Professionnelle, 52:424-445.

KETTLE, M., 1984. Disabled people and accidents. Journal of Occupational Accidents, 6:277-293.

LASSOW, G.; LEFFERS, P.; DE KROM, M . \& TRUST, J., 1997. Epilepsy in a Dutch working population: Are employees diagnosed with epilepsy disadventaged? Seizure, 6:95-98.

LITTORIN, M. E.; FEHLING, C.; ATTEWELL, R. G. \& SKERFVING, S., 1988. Focal epilepsy and exposure to organic solvents: A case-referent study. Journal of Occupational Medicine, 30:805-808. 
LONG, C. G. \& MOORE, J. R., 1979. Parenteral expectations for their epileptic children. Journal of Children Psychologic and Psychiatric, 20:299-312.

McLELLAN, D. L., 1987. Epilepsy and employment. Journal of the Society of Occupational Medicine, 37:94-99.

NIEDERMEYER, E., 1990. The Epilepsies: Diagnosis and Management. Baltimore: Urban \& Schwarzenberg.

OLSSON, I. \& CAM PENHAUSE, G., 1993. Social adjustment in young adults with absence epilepsies. Epilepsia, 34:846-851.

PENRY, K. J. \& DEVINSKY, A., 1993. Quality of life in epilepsy: The clinician's view. Epilepsia, 34 (Sup. 4):S4-S7.

PERRINE, K. R., 1993. A new quality-of-life inventory for epilepsy patients: Interim results. Epilepsia, 34 (Sup. 4):S4-S7.

RODIN, E., 1972. Medical and social prognosis in epilepsy. Epilepsia, 13:121-131.

RODIN, E., 1982. Epilepsy and work. In: A Textbook of Epilepsy (J. Laidlaw, A. Richens \& L. Osley, eds.), pp. 496-506, Edinburgh: Churchill Livingstone.

ROSS, D., 1988. Dealing with epilepsy. Occupational Health, 12:741-743.

RYAN, R.; KEMPNER, K. \& EMLEN, A., 1980. The stigma of epilepsy as a self-concept. Epilepsia, 21: 433-444.
SCAMBLER, G. \& HOPKINS, A., 1980. Social class, epileptic activity and disadvantage at work. Journal of Epidemiology and Community Health, 34: 129-133.

SCAMBLER, G., 1987. Sociological aspects of epilepsy. In: Epilepsy (A. Hopkins, ed.), pp. 503-506, Cambridge: Cambridge University Press.

SHAW, E. B., 1983. Resources avaible to the patient with epilepsy. In: Epilepsy: Diagnosis and Management (T. R. Browne \& R. G. Feldman, eds.), pp. 141-143, Boston: Little, Brown and Company.

TETTENBORN, B. \& KRÄMER, G., 1992. Total patient care in epilepsy. Epilepsia, 33 (Sup.1):S28-S32.

TRÖSTER, H., 1997. Disclose or Conceal ? Strategies of information management in persons with epilepsy. Epilepsia, 38:1227-1237.

UDEL, M. M., 1960. The work performance of epileptic in industry. Archives of Environmental Health, 1:91-98.

WALL, M.; BUCHANAN, N. \& BAIRD-LAMBERT, J., 1987. The management of epilepsy: Patients' perceptions and expectations. Medical Journal of Australia, 146:473-476.

WINGET, C. M.; HUGHES, L. \& LADOU, J., 1978. Physiological effects of rotational work shifting: A review. Journal of Occupational Medicine, 20:204210 